\title{
Percutaneous techniques for minimally invasive posterior lumbar fusion
}

\author{
Eric B. Harris, M.D., Patrick Massey, M.D., James Lawrence, M.D., \\ Jeffrey Rihn, M.D., Alex Vaccaro, M.D., Ph.D., AND D. Greg Anderson, M.D. \\ Department of Orthopaedic Surgery, Thomas Jefferson University, Philadelphia, Pennsylvania
}

\begin{abstract}
Percutaneous pedicle screw fixation for lumbar posterolateral instrumented fusion is an attractive alternative to standard open techniques. The technical aspects of this procedure can be challenging and even frustrating when first learning the technique. However, once these techniques have been mastered, they offer a safe, less invasive, less traumatic, more aesthetic method for performing fusion. The authors have outlined a step-by-step method for performing this surgery, and include a case series that demonstrates excellent results in patients treated with this procedure.
\end{abstract} (DOI: $10.3171 /$ FOC/2008/25/8/E12)

\section{KEY WoRdS • minimally invasive surgery - pedicle screw fixation • posterior lumbar fusion}

$\mathrm{M}$ INIMALLY invasive surgical techniques have been growing in popularity in recent years. Endoscopic techniques used by general surgeons, gynecologists, and orthopedists have become the standard of care for many conditions, and other minimally invasive surgical solutions are increasingly sought out by patients and their families.

Traditional techniques for posterior spinal fusion required large incisions, extensive stripping and retraction of the paraspinal musculature, significant postoperative pain, and long recovery times, which are believed to be, at least in part, due to damage to the erector spinae muscle group. ${ }^{5}$ The advent of pedicle screw fixation carried posterior fusion forward by providing more stable constructs, facilitating earlier mobilization, and allowing for better correction of deformity. ${ }^{1-4,6}$ The operative exposure, however, remained the same or even increased the surgical dissection and muscle damage necessary to perform a lumbar fusion with instrumentation compared with noninstrumented techniques. Follow-up MR imaging studies have demonstrated fatty degeneration of the paraspinal musculature after such exposures, which, in theory, could lead to worse clinical outcomes in terms of strength, pain scores, and lost work days. ${ }^{5}$

Minimally invasive techniques for posterior lumbar fusion use small-muscle splitting approaches to allow placement of hardware under fluoroscopic guidance. These

Abbreviations used in this paper: ODI = Oswestry Disability Index; PA = posteroanterior; VAS = visual analog scale; VB = vertebral body. approaches permit accurate hardware placement at multiple levels while avoiding the more extensive trauma associated with an open approach. In this paper we focus on a technique for percutaneous placement of cannulated pedicle screws and provide tips and tricks for fluoroscopy setup, surgical approach, pedicle cannulation, and screw insertion.

\section{Operating Room Setup}

After intubation and placement of neurophysiological monitoring equipment, the patient is positioned prone on a radiolucent Jackson table. Skin preparation and draping are performed as for an open procedure. The $\mathrm{C}$-arm mobile fluoroscopy unit is draped and brought into the field and true PA views of the levels of interest are obtained. When obtaining these views, proper alignment of the $\mathrm{C}$-arm is critical to the success of the procedure. A properly aligned PA image of a vertebra should show a flat superior endplate with no "double endplate shadow" visible. The pedicles should appear just below the superior endplate, and the spinous processes should be centered between the pedicles. When imaging the lower lumbar spine, it is important to use the Ferguson view to obtain these images. It is important to note that each level will generally have a slightly different sagittal plane orientation, and the exact Ferguson angulation of each level should be noted and marked on the $\mathrm{C}$-arm to assist the radiology technician in quickly returning to a level when this is requested during the procedure.

On the lateral image, the superior endplate should again appear flat and the pedicles should be superimposed. The 


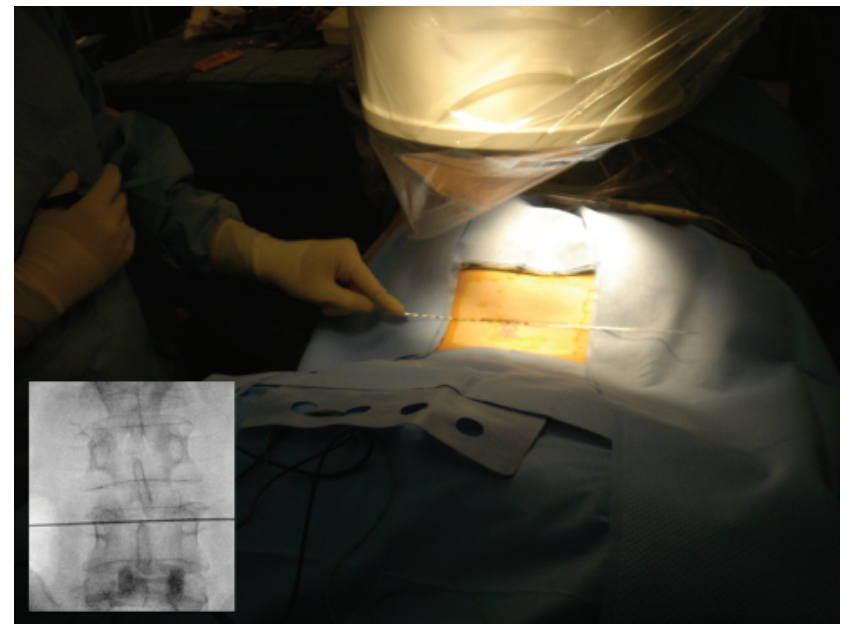

FIG. 1. Preoperative photograph and radiographic image (inset) showing placement of a guidewire on the skin for proper marking of the craniocaudad location of the pedicles undergoing instrumentation.

posterior cortex of the VB should only show a single shadow when rotation of the segment has been eliminated. The lateral aspects of both pedicles should be visible and superimposed on a true lateral view. If proper fluoroscopic views cannot be adequately obtained because of a patient's severe obesity, shadowing from intraabdominal air, or severe osteopenia, percutaneous screw placement should not be attempted unless more advanced imaging modalities such as 3D CT or computer navigation systems are available. Discussion of the utility and technical aspects of such modalities is beyond the scope of this paper.

Once an adequate PA fluoroscopic image has been obtained, a wire is placed on the skin and the location of bone landmarks is drawn on the skin with a surgical marker. Specifically, lines should be drawn through the centers of the pedicles (intrapedicular line) at each level on a properly aligned PA view. Next, lines are drawn in a cephaladcaudad orientation $\sim 1 \mathrm{~cm}$ lateral to the lateral wall of the pedicles to be instrumented (Figs. 1 and 2). We prefer to mark each line through the center of the pedicles with a label indicating the level and to note the exact sagittal plane orientation of the $\mathrm{C}$-arm to obtain the properly aligned image.

\section{Surgical Technique}

The skin incision is made along the cephalocaudad line between the upper and lower intrapedicular line when performing instrumentation of the lower lumbar region. For the L3-S1 levels, a single 1-1.5 inch incision usually suffices because the normal lordosis of the spine allows the surgeon to reach all those levels easily through the single incision. The fascia is incised and opened underneath the skin edges slightly with scissors because it is less mobile than the skin and may make maneuvering within the small incision difficult. Blunt finger dissection is used to gently divide the paraspinous muscle between the fascicles down to the junction between the base of the transverse process and the lateral aspect of the facet joint. It is quite simple, with practice, to palpate the ideal starting point for pedicle

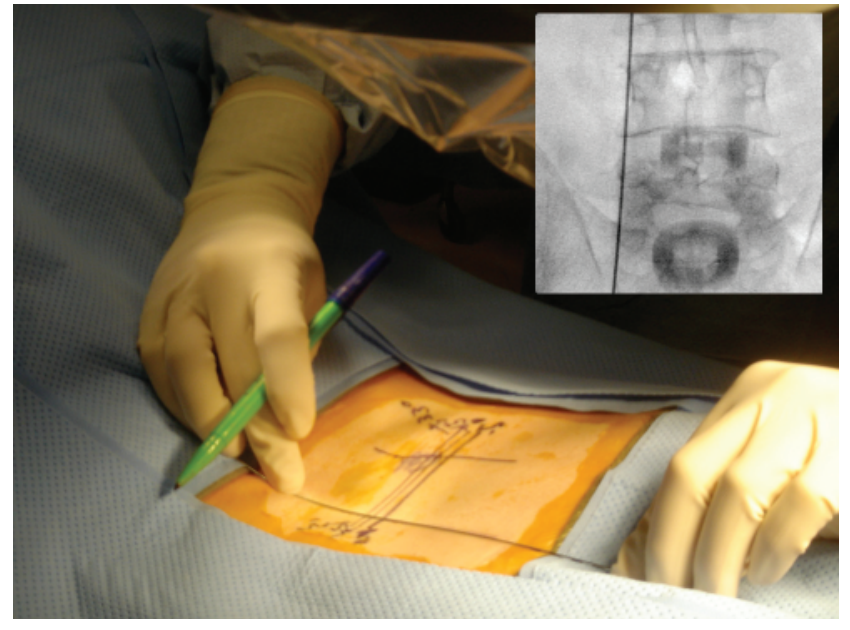

FIG. 2. Preoperative photograph and radiographic image (inset) showing placement of a guidewire on the skin for proper marking of the lateral border of the pedicles undergoing instrumentation. A line should be drawn on the skin $1 \mathrm{~cm}$ lateral to this wire, and the skin incision should be made at this location.

cannulation, which helps when docking the Jamshidi needle without multiple fluoroscopic images. If indicated, it is also relatively simple to use a tubular retractor system in this incision to decorticate the transverse processes and facet and deploy bone graft for an intertransverse onlay type of fusion. If this type of fusion is done, it should be completed prior to the placement of pedicle screws, which will block access to the space.

Using a Jamshidi needle, docked against the bone at the junction of the base of the transverse process and facet joint, a PA image is obtained to localize the tip of the needle. The correct docking point is directly over the lateral wall of the pedicle, at the 3-o'clock site on the right side and the 9-o'clock site on the left side. If necessary, the tip of the Jamshidi needle should be adjusted until it is correctly positioned prior to proceeding. Once correctly placed over the lateral wall, the needle is gently tapped with a mallet to engage the needle tip in the bone and keep it from slipping. The Jamshidi needle should be angled about $10^{\circ}$ from lateral to medial according to the normal orientation of the pedicle axis in the transverse plane. Another PA image is obtained to document correct positioning of the needle tip and also to scrutinize the orientation of the needle through the pedicle. The shaft of the needle should be seen to be parallel with the endplate. The surgeon can easily ascertain from the angle of the shaft of the needle and the endplate whether the needle is aiming colinear with the pedicle or is aiming either upward or downward along the pedicle axis. With the needle tip slightly embedded $(<5$ $\mathrm{mm}$ ) in bone and the shaft of the needle correctly aligned with the axis of the pedicle, a marking pen is used to draw a line on the needle $\sim 20 \mathrm{~mm}$ above the skin. This mark serves as a guide for the surgeon as to the depth of the needle tip as it is passed through the pedicle. When the marked line reaches the skin edge, the tip of the needle should be at the junction of the pedicle and VB on a lateral fluoroscopic view. The needle is then slowly advanced with gentle taps of a mallet through the pedicle until it reaches the 20$\mathrm{mm}$-depth line mark. Intermittent fluoroscopic images can 


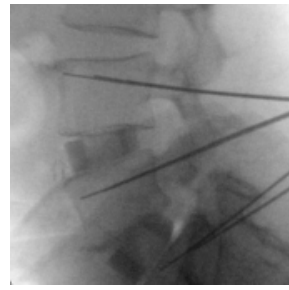

FIG. 3. Lateral radiograph demonstrating satisfactory placement of guidewires in the pedicles.

be taken as needed while traversing the pedicle to monitor the progress of the needle.

Once the depth marking has reached the skin edge, a PA image is taken. At this point, the tip of the needle should appear to have traversed the pedicle from lateral to medial and should be no more than three-quarters of the distance from lateral to medial across the pedicle on the PA view. The needle trocar may then be removed and a flexible guidewire placed. Gentle pressure may be used to advance the guidewire 1-2 cm into the cancellous bone of the VB, and the Jamshidi needle may then be removed, taking care to maintain positive control of the guidewire at all times throughout the remainder of the procedure. The other pedicles are prepared in a similar fashion, until guidewires have been placed in all desired pedicles. Using this technique, PA fluoroscopy can be used primarily during pedicle cannulation. On completion of cannulation, a lateral fluoroscopic image is obtained to verify correct placement of all guidewires (Fig. 3). Tactile feedback plays an important role in the percutaneous cannulation of pedicles. The bone of the pedicle is relatively soft and should allow smooth advancement of the needle. If hard "cortical" bone is reached and the needle does not advance easily, chances are good that the needle is too medial, has entered the facet joint, and is striking the superior articular process or even the cortical wall of the pedicle. In such a situation, the surgeon should stop and assess the situation with fluoroscopy and readjust the needle position prior to proceeding.

Once the guidewires are in place, we find it easiest to place the C-arm in a cross-table lateral projection. Soft-tissue dilators are placed over each guidewire to protect surrounding soft tissue during preparation of the pedicles. Pedicles are tapped using cannulated taps. Once the tap has reach the base of the pedicle, it can be stimulated using stimulus-evoked electromyography to ensure that there is no low-voltage activity to raise concern of a pedicle wall breech. Throughout pedicle preparation, the surgeon must at all times maintain 2 fingers on the guidewire to ensure that it neither advances nor is inadvertently pulled out as the tap is withdrawn. We find it easiest to remove the handle of the tap to allow the surgeon to withdraw the device with constant control of the guidewire. Cannulated pedicle screws are then inserted with their attached screw extensions, which facilitate rod passage. It is important to align the screw heads carefully in a cephalocaudad direction to ensure proper passage of the rod. Once this alignment is satisfactory, the rod may be passed using the technique specific to the pedicle screw system used. The construct may be compressed or distracted as appropriate prior to final tightening, and screw extensions are then removed and final radiographs may be obtained (Fig. 4).

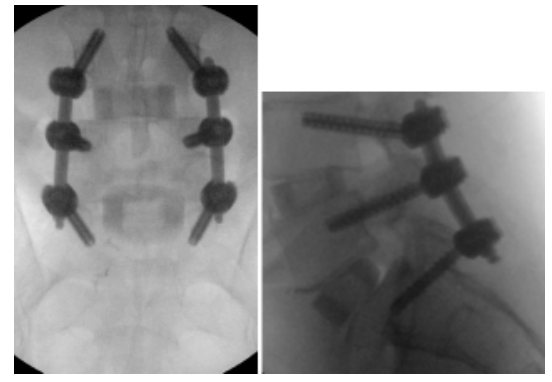

FIG. 4. Final PA (left) and lateral (right) radiographs showing the completed pedicle screw and rod construct.

\section{Case Series}

Twenty-nine consecutive patients underwent 1- or 2level posterolateral percutaneous instrumented fusion for symptomatic spondylolisthesis according to the methods described earlier. The figures used to illustrate the technique are from a case not included in the series. All operations were performed by the senior surgeon (D.G.A.). Patients completed VAS and ODI testing preoperatively, at the 3-month, and at the 12-month follow-up time points. The mean age was 65 years, and there were 19 female and 10 male patients in this group.

The surgical procedure consisted of a decompression of the neural elements at the site of the spondylolisthesis, posterolateral onlay fusion, and instrumentation. The mean surgical duration for all patients was 141 minutes (range 90-300 minutes). The mean blood loss for all patients was $222 \mathrm{ml}$ (range 50-600 ml). The mean length of stay for these patients was 2.48 days (range $1-5$ days).

The mean pain score as assessed using the VAS was 7.8 (range 5-10) preoperatively, 2.9 (range 0-8) at 3 months, and 2.2 (range $0-5$ ) at 12 months (Table 1). Functional outcome, as assessed using the ODI, improved from severe disability (mean score 46 of 100) preoperatively, to moderate disability (mean score 27 of 100) at 3 months, to minimal disability (mean score 14 of 100) at 12 months postoperatively.

Fusion of the constructs at the 12-month time point was defined as an absence of lucency around the hardware, with continuous bone graft consolidation bridging the transverse processes of the fused level on the PA and lateral x-ray studies, and no apparent motion on flexion and extension $\mathrm{X}$ ray studies of the lumbar spine. Using these criteria, successful fusion of the constructs was found in all but 1 patient. In the case in which fusion did not occur, the

TABLE 1

Pain and disability scores in 29 patients who underwent 1- or 2-level posterolateral instrumented fusion for symptomatic spondylolistheseis

\begin{tabular}{cccc}
\hline \hline & \multicolumn{3}{c}{ Score* } \\
\cline { 2 - 4 } Scale & Preop & 3 Mos & 12 Mos \\
\hline VAS & $7.8(5-10)$ & $2.9(0-8)$ & $2.2(0-5)$ \\
ODI & $46(12-80)$ & $27(0-58)$ & $14(0-60)$ \\
\hline
\end{tabular}

* Values represent the mean pain scores on the VAS ( 0 is minimum, 10 is maximum) and the mean functional restriction score on the ODI (scores range from 0 to 100 of 100$)$. The range is given in parentheses. 
patient had evidence of haloing around the L-5 screw, with lack of fusion between L-4 and L-5. However, the patient has not required revision surgery for clinical symptoms.

There was 1 perioperative complication; 1 patient had a small dural tear, which was successfully repaired intraoperatively with no sequelae. There was 1 postoperative complication, a deep venous thrombosis of the calf that was successfully treated with Coumadin.

Previously published papers have shown that patients treated for spondylolisthesis with open instrumented fusion have significant improvement in disability scores and pain compared with their nonsurgically treated counterparts. ${ }^{7,8}$ Our series showed improvement in disability and pain that was similar to findings in these reports. Möller and Hedlund showed improvement in pain scores from a mean of 63 to 36, whereas our patients improved from a mean score of 7.8 to 2.2. Additionally, Möller and Hedlund's patients saw an improvement in disability scores from 44 to 30 based on the Disability Rating Index, whereas our patients' ODI scores improved from 46 to 14 . Blood loss in our series was significantly lower, at a mean of 222 versus $1517 \mathrm{ml}$ in the Möller study, and the operative time was similarly improved, at a mean of 141 minutes compared to 298 minutes. Our results mirror the positive outcomes seen in previous studies and, we believe, may improve on them due to presumably decreased morbidity from shorter anesthesia times and less blood loss.

\section{Conclusions}

Posterolateral instrumented fusion with pedicle screw constructs is the gold standard for treatment of symptomatic spondylolisthesis. Historically, patients undergoing this operation have good to excellent outcomes with traditional surgical techniques, and thus this patient cohort allows for comparison of outcomes by using a less invasive surgical strategy. Concern over the approach-related morbidity caused by excessive paraspinal muscle stripping, combined with a general trend throughout most surgical specialties toward less invasive techniques, has led to the development of several minimally invasive pedicle screw systems. Utilization of percutaneous techniques allows smaller incisions, less muscle stripping, lower blood loss, and excellent fusion rates and clinical outcomes. ${ }^{9}$ The drawbacks to this technique include the inherent learning curve, increased exposure to ionizing radiation, and increased operating times compared to an open surgical approach. Once the concepts and techniques of this procedure have been mastered, however, it offers a less traumatic, more aesthetic, and equally effective method for posterolateral fusion.

\section{References}

1. France JC, Yaszemski MJ, Lauerman WC, Cain JE, Glover JM, Lawson KJ, et al: A randomized prospective study of posterolateral lumbar fusion. Outcomes with and without pedicle screw instrumentation. Spine 24:553-560, 1999

2. Glaser J, Stanley M, Sayre H, Woody J, Found E, Spratt K: A 10year follow-up evaluation of lumbar spine fusion with pedicle screw fixation. Spine 28:1390-1395, 2003

3. Hamill CL, Lenke LG, Bridwell KH, Chapman MP, Blanke K, Baldus $\mathrm{C}$ : The use of pedicle screw fixation to improve correction in the lumbar spine of patients with idiopathic scoliosis. Is it warranted? Spine 21:1241-1249, 1996

4. Kanayama M, Cunningham BW, Sefter JC, Goldstein JA, Stewart G, Kaneda K, et al: Does spinal instrumentation influence the healing process of posterolateral spinal fusion? An in vivo animal model. Spine 24:1058-1065, 1999

5. Kim DY, Lee SH, Chung SK, Lee HY: Comparison of multifidus muscle atrophy and trunk extension muscle strength: percutaneous versus open pedicle screw fixation. Spine 30:123-129, 2005

6. Liljenqvist U, Lepsien U, Hackenberg L, Niemeyer T, Halm H: Comparative analysis of pedicle screw and hook instrumentation in posterior correction and fusion of idiopathic thoracic scoliosis. Eur Spine J 11:336-343, 2002

7. Möller H, Hedlund R: Instrumented and noninstrumented posterolateral fusion in adult spondylolisthesis-a prospective randomized study: part 2. Spine 25:1716-1721, 2000

8. Möller H, Hedlund R: Surgery versus conservative management in adult isthmic spondylolisthesis-a prospective randomized study: part 1. Spine 25:1711-1715, 2000

9. Park Y, Ha JW: Comparison of one-level posterior lumbar interbody fusion performed with a minimally invasive approach or a traditional open approach. Spine 32:537-543, 2007

Manuscript submitted My 15, 2008.

Accepted June 2, 2008.

Sources of support: none reported.

Address correspondence to: Eric B. Harris, M.D., Department of Orthopaedic Surgery, Thomas Jefferson University, 925 Chestnut Street, 5th Floor, Philadelphia, Pennsylvania 19107. email: ericbharris@gmail.com. 